\title{
Rare intradural extramedullary spinal granular cell tumor in a young female
}

\author{
Abstract \\ Granular cell tumor (GCT) is a rare tumor, usually benign with characteristic \\ histomorphology. Location of GCT varies widely within the body, but the spine is clearly \\ a rare location for GCT. We report a rare case of granular cell tumor involving intradural \\ extramedullary portion of thoracolumbar region of spinal cord. Knowledge of GCT is \\ important as subsequent prognosis differs from other tumor at same location. GCTs are \\ resistant to chemotherapy and radiotherapy, but our patient responded well with no disease \\ in one year follow up. \\ Keywords: granular cell tumor, intradural, spinal cord, radiotherapy
}

Volume 7 Issue 3 - 2018

\author{
Abhishek Soni,' Nupur Bansal \\ 'Department of Radiation Oncology, Kainos Super Speciality \\ Hospital, India \\ ${ }^{2}$ Department of Radiation Oncology, Max Super Speciality \\ Hospital, India
}

Correspondence: Abhishek Soni, Department of Radiation
Oncology, Kainos Super Speciality Hospital, Rohtak, Haryana,
India,Tel +9I-8708997069, Email abhisheksoni246@gmail.com

Received: May II, 2018 | Published: June 29, 2018

\section{Introduction}

Granular cell tumor (GCT) is one of the rare neoplasms and it is called GCT due to its nest like arrangement of polyhedral cells with abundant eosinophilic granular cytoplasm. Most common site for GCT is head and neck (particularly tongue) and skin. GCT can affect any body site virtually but breast; respiratory tract and gastrointestinal tract are some other predominant sites. ${ }^{1,2}$ Intradural extramedullary spinal GCT is exceptionally rare presentation. We present here a rare case of GCT in intradural extramedullary region of thoracolumbar spinal cord.

\section{Case report}

An eleven years old female presented with complaints of headache, vomiting and backache. Headache was sudden onset, of severe intensity, global and was relieved by analgesics. On examination, vitals were within normal limits and systemic examination revealed stiff neck, decreased bilateral plantar reflexes, positive kernig and brudzinski sign. NCCT head was normal. Then patient developed hypertension. In view of suspicion of chronic meningitis, ATT was started and lumbar puncture was performed. On examination CSF was grossly hemorrhagic, TLC $100 / \mathrm{cmm}$, lymphocytes $85 \%$. Patient was put on acyclovir in view of hemorrhagic CSF. T2 sagittal CEMRI whole spine (Figure 1) \& (Figure 2) revealed isointense, oval, well-defined, $2.5 \times 3 \mathrm{~cm}$ mass in anterior intradural space with multiple T2 hypointense / hyperintense foci with free blood collection in subarachnoid space anteriorly. Spinal cord proximal to D12/ L1 vertebrae level appeared displaced dorsally with widening of subarachnoid space anteriorly with adhesions. T1 sagittal MRI showed isointense signal of mass with same features. Spinal leptomeninges proximally as well as distally were enhanced. CEMRI brain revealed leptomeningeal enhancement of brain. A diagnosis of spinal and cerebral SAH was made with homogenously enhancing intradural mass lesion at D12/L1 level suspicion of Schwannoma, with SAH originating from Schwannoma. Patient condition got deteriorated day by day in between, and she started to develop upper motor neuron signs in left lower limb which progressed to right lower limb also. She developed bilateral lateral rectus palsy and reported decreased vision. Then she got operated by neurosurgeon and D12 laminectomy with near total microdissection of anterior cord tumor was performed. In post-operative period, patient continued to have features of raised intracranial tension, stiff neck, paraplegia, hypertension and vision deterioration. PCR for HSV was negative. NCCT head was normal. Histopathology revealed features of Schwannoma with extensive hemorrhage. IHC was negative for GFAP, and diffusely positive for S-100, suggestive of Schwannoma. Post operative MRI (Figure 3) showed near complete excision of Schwannoma with post-operative leptomeningeal dural enhancement at D12/L1 vertebral level with resolving intradural hemorrhage and evidence of post hemorrhagic arachnoiditis. MRI brain revealed dilated ventricular system (?Post hemorrhagic) with frontal horn dimension of $1 \mathrm{~cm}$, and diagnosis of hydrocephalus was made. For hydrocephalus, ventriculoperitoneal shunt procedure (Figure 4) was performed and her condition got improved, but patient continued to remain hypertensive. USG abdomen with renal artery Doppler was normal, but urinary VMA levels were elevated. Ophthalmoscopy revealed slight blurring of margins of fundus, bilateral disc pallor temporally more in the left side eye, suspicious of left optic atrophy. PCR for tuberculosis was negative, CSF ADA was normal. ATT was stopped after one week. Acyclovir was stopped after MRI evidence of SAH. IV antibiotics and DVT prophylaxis and supportive medications were continued. She was operated again for residual intradural spinal tumor at D12/ L1 vertebrae level. Histopathology revealed granular cell tumor at D12/L1 vertebral level. Sections revealed sheets and nests of large and polygonal cells, with abundant eosinophilic, granular cytoplasm; small, dark staining nucleus, and small, indistinct nucleolus. No nuclear atypia or abnormal mitotic activity was observed.

On immunohistochemistry, the tumor cells were strongly positive for S-100, many cells were positive for CD 68, negative for SMA, and Mib-1 labelling index was low (1-2\%). CT head showed VP shunt in right lateral ventricle with mild dilatation of lateral and third ventricle. After that, she presented with complaints of weakness in lower limbs and she developed hypertension and urinary retention. MRI LS spine revealed post op status at D12-L1 level with postoperative arachnoiditis with clumping of nerve roots of cauda equina with compression of lower end of spinal cord and conus. Urodynamics study revealed neurogenic bladder (atonic bladder). MRI dorso lumbar spine with contrast showed arachnoiditis as persisting clumping of 
cauda equine nerve roots with loculated CSF compressing the lower spinal cord and conus medullaris, displacing it posteriorly; nodular enhancing thickening at D12 level anterior to cord, mild reduced anterior disc height at D12-L1 with mild kyphotic deformity.

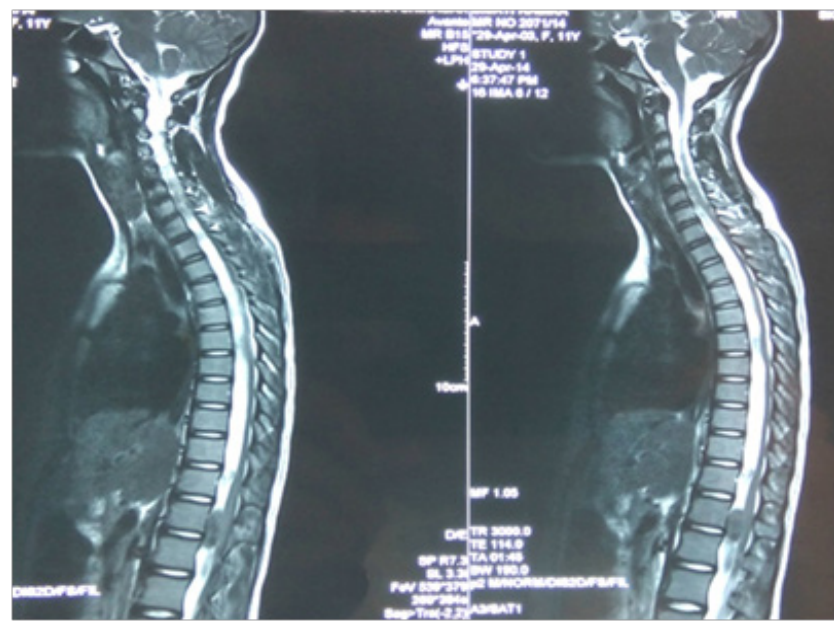

Figure I T2 weighted MRI image revealing mass in anterior intradural space.

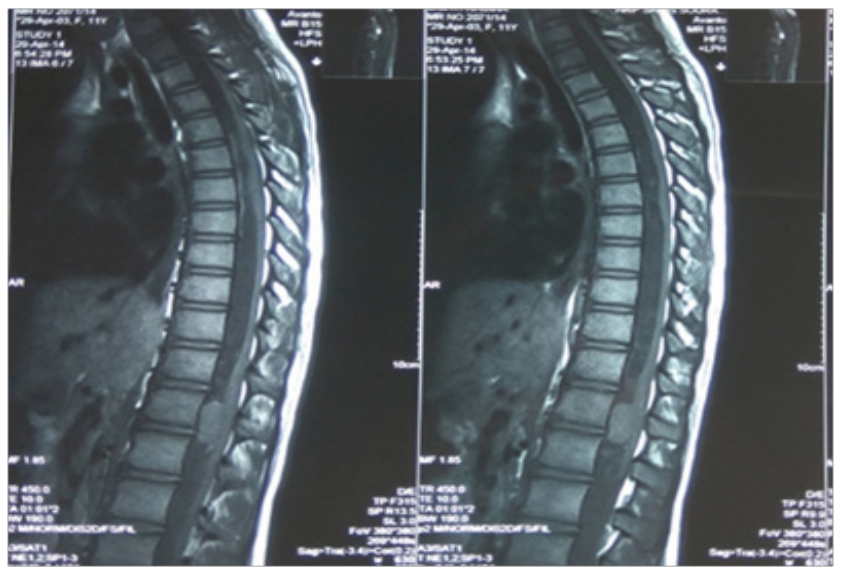

Figure 2 TI weighted MRI showing mass at TI2-LI level.

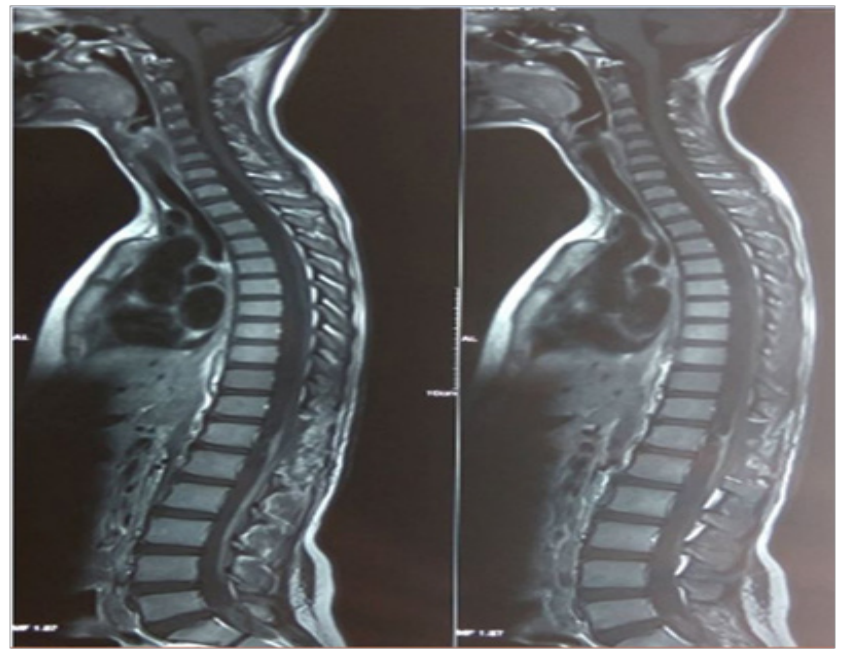

Figure 3 Post operative MRI showing near complete excision of tumor with leptomeningeal dural enhancement at DI2/LI vertebral level.

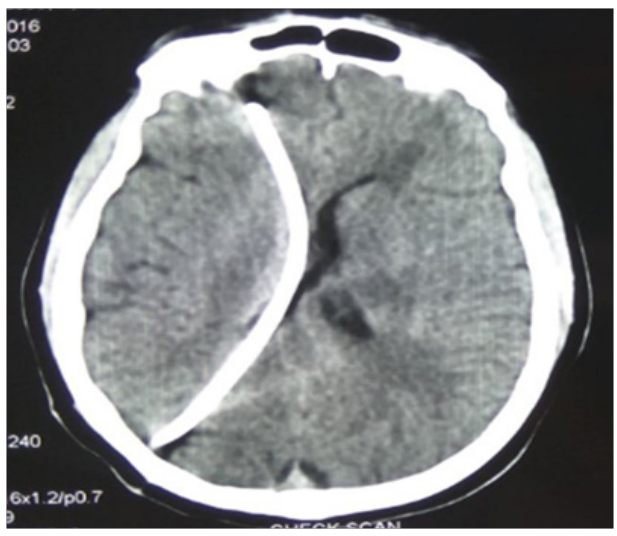

Figure $4 \mathrm{CT}$ showing ventriculo-peritoneal shunt.

After a short follow up time, she presented again with complaints of fever, headache and painful movement of neck. MRI of thoracolumbar spine revealed dural mass lesion with uniform enhancement on the post-contrast T1fat-sat images. There was also enhancement of the dural sheath superior and inferior to the lesion. That most likely represented recurrent Schwannoma with meningioma as differential diagnosis. She underwent D12-L1 exploration and excision of intradural extramedullary tumor and part of the tumor attached to cord / nerve roots were left behind. Histopathology revealed intradural spinal recurrent granular cell tumor at D12/L1 vertebral level (Figure 5) and (Figure 6). Sections revealed a cellular tumor composed of loose and tight sheets of large, round to polygonal cells, with scanty amount of intervening stroma. The tumor cells revealed copious amount of bright granular eosinophilic cytoplasm, and centrally placed round nuclei showing mild pleomorphism, prominent nucleoli and vesicular chromatin. Cytologic atypia, mitoses or necrosis were not seen within the tumor. But again she presented with back pain after short period.

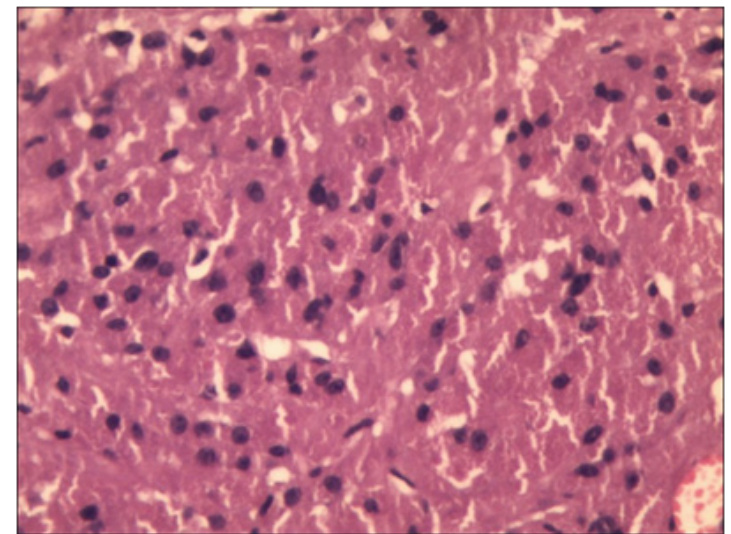

Figure 5 Sheet of large round to polygonal closely packed tumor cells with abundant granular eosinophilic cytoplasm (H\&E 200x).

CEMRI of dorso-lumbar spine demonstrated evidence of laminectomy at D11, D12 and L1 vertebrae levels with associated post operative changes in the form of soft tissue posteriorly and irregular linear hypointensities, with CSF loculations posterior to D12 vertebral body. A well-defined eccentric intradural nodular area of heterointensity with enhancement was seen anterior to the cord in midline and to the left at D12 vertebral level, suggestive of residual tumor. The cord was displaced right posterolaterally. There was 
mild thickening with clumping of the nerve roots of cauda equina along the periphery of the thecal sac along with diffuse thickening and enhancement along the anterior epidural space extending from D12-L1 to L5-S1 levels - represented changes of arachnoiditis. Diffuse increased intramedullary signal alteration showed hyperintensity on $\mathrm{T} 2 \mathrm{~W}$ images in the cervicodorsal cord with mild subtle expansion of the cervical cord rostral to the operative site, and altered CSF flow in the anterior spinal subarachnoid space, possibly suggestive of presyrinx formation. Mild anterior wedging of D11/D12 noted with reduced focal kyphotic angulation. Then she was planned for radiation treatment and a rigid deformable thermoplastic mask was made for day to day immobilization. Radiation planning CT and MRI were registered and fused. The GTV (gross tumor volume) was marked after CT-MRI registration as the gross disease visible. CTV (clinical target volume) included whole of the spinal canal and one $\mathrm{cm}$ cranio-caudal margin was taken for microscopic extension, and it was trimmed from the bony portions. PTV (planning target volume) was delineated as $0.5 \mathrm{~cm}$ margin from CTV. OARs (organs at risk) were marked as spine, PRV (planning organ at risk volume) spine, kidneys, bowel bag, lungs, heart, liver and esophagus; and their dose constraints were respected. Rapid Arc plan was generated on linear accelerator using $6 \mathrm{MV}$ photons. She received 39.6 Gy in 22 fractions at 1.8 Gy per fraction (Figure 7), then boost was planned with the minimal margins (approximate $5 \mathrm{~mm}$ ) around GTV for PTV boost. She received 5.4 Gy in 3 fractions with PTV boost (Figure 8). Thus, she received 45 Gy in 25 fractions over 5 weeks. She tolerated the radiation well. Follow-up MR imaging performed 6 months after surgery plus radiation showed no evidence of recurrence.

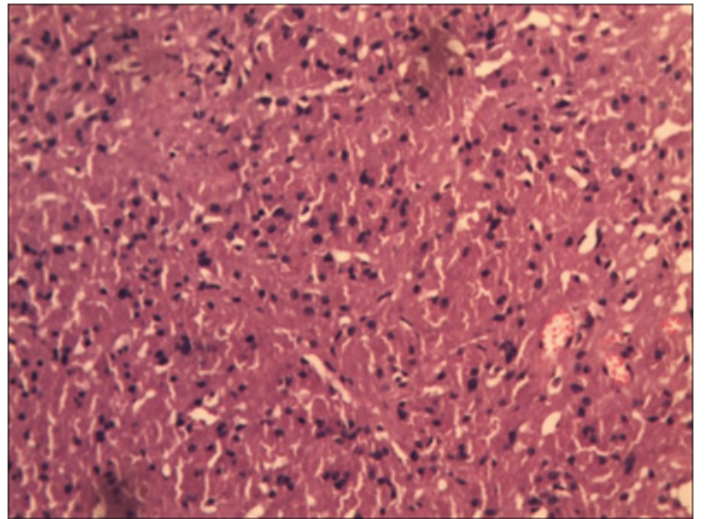

Figure 6 These cells have small round central to eccentrically placed vesicular nuclei with no nuclear atypia (H\&E 400x).

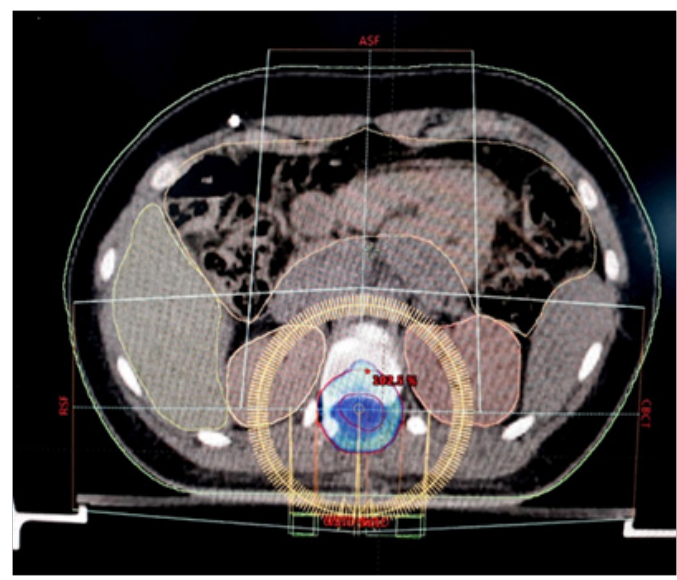

Figure 7 Dose colour wash display showing dose received by the PTV (outer red circle) with Dmax as $102.5 \%$ of the prescribed dose; OAR delineated as liver, bowel bag, spine, left and right kidney.

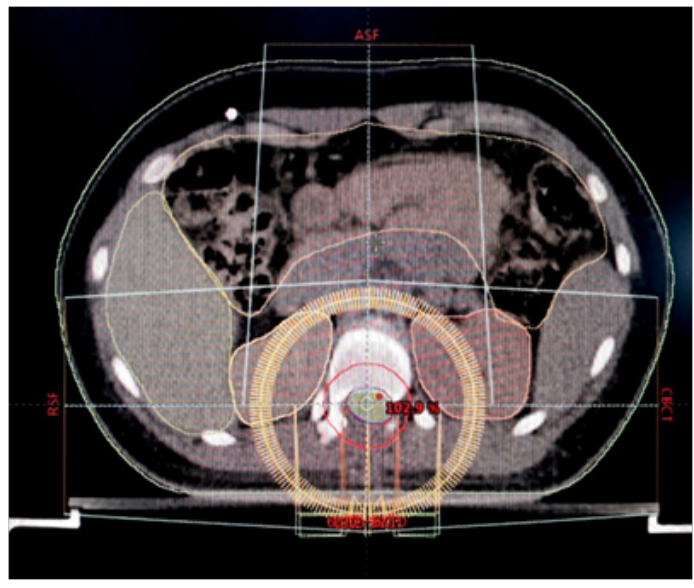

Figure 8 Dose colour was display for PTV boost region (inner red circle) with Dmax as $102.9 \%$ of the prescribed dose.

\section{Discussion}

Granular cell tumor (GCT) is one of the rare neoplasms and it is called GCT due to its nest like arrangement of polyhedral cells with abundant eosinophilic granular cytoplasm. Abrikossoff first described GCT as granular cell myoblastoma. ${ }^{1}$ Granular cell tumors (GCTs) are thought to originate from neural tissue based on immunohistochemical features. GCTs can affect any part of the body but are most commonly found in the skin, head and neck (particularly tongue), brain, ovaries and breast tissue. ${ }^{1,3-5}$ Other predominant sites include breast, respiratory tract and gastrointestinal tract; however, virtually any body site may be affected. ${ }^{2}$ But GCT of the spine, with intradural extramedullary location, is exceptionally rare. Granular cell tumors in the central nervous system (CNS) region was reported in the pituitary stalk, neurohypophysis and spinal intradural location, $3,6,7$ and approximate 50 cases has been reported till now since the first documentation in $1893 .{ }^{8} \mathrm{GCT}$ of the spine usually presents in 13 to 49 year age range, but the median age of presentation is 23 years, and striking preponderance for females. Clinical features include radicular symptoms, backache, and slowly progressive neurological findings like weakness, impotence, paresthesia, and problems with bowel and bladder function, gait, upper and lower motor neuron dysfunction etc. ${ }^{69-12}$ These tumors usually spread via hematogenous and lymphatic and routes to the liver, lungs, and skeletal structures. ${ }^{13}$

GCTs are usually benign tumors, and malignant features are seen in only $1-10 \%$ of GCTs. ${ }^{14}$ Malignant GCT is usually diagnosed by a combination of clinical malignant behavior and histological findings, including increased mitotic activity, cellular pleomorphism and necrosis. If the primary lesion is large sized or rapidly growing, or if there is evidence of distant metastases; clinical malignant lesion should be highly suspected. ${ }^{2,14}$ However, the rate of recurrent disease for benign GCTs is $20 \%$, and that of malignant GCTs is above $30 \%$. Thus, it is vital to differentiate GCTs from some other benign tumors like Schwannomas because of totally different clinical courses and outcomes. ${ }^{5}$ Immunohistochemistry and electron microscopy have demonstrated that GCT show Schwannian differentiation. Still, pathogenesis of spinal GCT is still controversial. ${ }^{12,15}$ Some authors reported that GCT of CNS region arise from Schwann cells, and other authors suggested that these arise from pituicyte (modified astrocyte) ${ }^{6,8,16-20}$ Lee et al., ${ }^{21}$ reported that spinal GCT derive not only from Schwann cells but also from tumor cells and degenerated normal cells. ${ }^{21}$ 
MRI is the investigation of choice. Typically the spinal GCTs are slightly hypointense on the T1-weighted sequences, and reveal homogenous contrast enhancement after intravenous Gadolinium injection. T2-weighted sequences of GCT typically show a hypointense signal. But the MRI tissue characteristics are not specific for GCT, because same characteristics can be seen in some of the other spinal canal tumors like meningioma, Schwannoma, ependymoma, paraganglioma or metastatic tumor. ${ }^{2,6,9-11}$ Some of the GCTs show speckled dots in the primary tumor region characterized by the low signal dots, particularly on enhanced T1-weighed sequences which is useful in diagnosis of GCT. ${ }^{5}$ GCT in subdural space must be differentially diagnosed from Schwannoma and meningioma. GCT is hard to distinguish from meningioma if calcification is absent. Characteristic feature of Schwannoma (nerve sheath tumor) is the appearance of dumbbell-shaped lesion with extraforaminal and intraforaminal components. GCT is difficult to differentiate from Schwannoma if the later presents with a solitary lesion. ${ }^{6}$ The recommended treatment of choice for spinal GCT is excision with wide margins. ${ }^{2}$ Wide local excision is the treatment of choice for benign GCTs, still, rates of recurrence is $2-8 \%$ when margins are negative and $>20 \%$ when margins are positive. ${ }^{13,22}$ Ordonez reviewed 41 cases of malignant GCTs, most of them were managed with wide local excision, and rate of recurrence was found to be $59 \% \cdot{ }^{13,23}$ Benign or malignant nature of GCT defines the prognosis of the tumor. Chemotherapy and radiotherapy are controversial because of the resistance of GCT towards them. ${ }^{13,22}$ Chemotherapy and radiotherapy was used in the past when malignant GCT was present or complete excision of GCT with wide margins was not possible, but their effectiveness is still to be proven. Although, only few intraspinal GCT are reported till date, radiation treatment has been successfully used to stabilize residual or recurrent disease in only one of the case., ${ }^{2,11}$

Around ten cases have been documented of GCT at this rare site, so the literature regarding radiotherapy at GCT spine is extremely lacking. No recommended dose regimen is available. But taking consideration the spine, the tumor was targeted to receive 45 Gy at conventional dose fractionation. ${ }^{5}$ In nutshell, treatment for spinal GCT has largely been unsatisfactory, particularly for the metastatic disease. Early recognition of spinal GCT as well as differential diagnosis of GCT like tumors in the settings of atypical presentation, must be taken into careful consideration as well, because spinal GCT can transform into malignant GCT and can be refractory to the conventional treatment and radiation may be helpful to decrease the recurrence rate as was seen in our case. ${ }^{13}$

\section{Acknowledgements}

None.

\section{Conflict of interest}

Author declares that there is no conflict of interest.

\section{References}

1. Ordóñez NG, Mackay B. Granular cell tumors: A review of the pathology and histogenesis. Ultrastruc Pathol. 1999;23(4):207-22.

2. Vaghasiya VL, Nasit JG, Parikh PA, et al. Intradural spinal granular cell tumor. Asian J Neurosurg. 2014;9(2):96-8.

3. Markesbery WR, Duffy PE, Cowen D. Granular cell tumors of the central nervous system. J Neuropathol Exp Neurol. 1973;32:92-109.
4. Rhee DJ, Choi YL, Suh YL, et al. Atypical granular cell tumor of the sellar region. J Korean Neurosurg Soc. 2006;40(6):459-62.

5. Lee CH, Hyun SJ, Lee JW, et al. Granular Cell Tumor of the Intradural Extramedullary Spinal Cord: Report of Two Cases with Respect to Radiological Differential Diagnosis. $J$ Korean Neurosurg Soc. 2013;53(2):121-4.

6. Qu J, Ma J, Luo L, et al. Subdural granular cell tumor in thoracic vertebral canal. Neurol India. 2009;57(5):679-81.

7. Strömblad LG, Brun A, Cameron R, et al. spinal granular cell tumor with subarachnoid hemorrhage: Case report. Neurosurgery. 1987;21(2):2303 .

8. Buhl R, Hugo HH, Hempelmann RG, et al. Granular cell tumour: A rare suprasellar mass. Neuroradiology. 2001;43(4):309-12.

9. Takayama Y, Hasuo K, Takahashi N, et al. Granular cell tumor presenting as an intradural extramedullary tumor. Clin Imaging. 2004;28(4):271-3.

10. Critchley GR, Wallis NT, Cowie RA. Granular cell tumour of the spinal cord: Case report. Br J Neurosurg. 1997;11(5):452-4.

11. Burton BJ, Kumar VG, Bradford R. Granular cell tumour of the spinal cord in patient with Rubenstein-Taybi syndrome: case report. $\mathrm{Br} J$ Neurosurg. 1997;11(3):257-9.

12. Weinstein BJ, Arora T, Thompson LD. Intradural, extramedullary spinal cord granular cell tumor: A case report and clinicopathologic review of the literature. Neuropathology. 2010;30(6):621-6.

13. Kim YI, Lee CK, Cho KH, et al. Granular Cell Tumor of Brachial Plexus Mimicking Nerve Sheath Tumor: A Case Report. Korean J Spine. 2012;9(3):275-7.

14. Fanburg-Smith JC, Meis-Kindblom JM, Fante R, et al. Malignant granular cell tumor of soft tissue: diagnostic criteria and clinicopathologic correlation. Am J Surg Pathol. 1998;22(7):779-94.

15. Rickert $\mathrm{CH}$, Kuchelmeister K, Gullotta F. Morphological and immunohistochemical characterization of granular cells in non-hypophyseal tumours of the central nervous system. Histopathology. 1997;30(5):464-71.

16. Kurtin PJ, Bonin DM. Immunohistochemical demonstration of the lysosome-associated glycoprotein CD68 (KP-1) in granular cell tumors and schwannomas. Hum Pathol. 1994;25(11):1172-8.

17. Nathrath WBJ, Remberger K. Immunohistochemical study of granular cell tumours: Demonstration of neuron specific enolase, S-100 protein, laminin and alpha-1-antichymotrypsin. Virchows Arch a Pathol Anat Histopathol. 1986;408(4):421-34.

18. Schaller B, Kirsch E, Tolnay M, et al. Symptomatic granular cell tumor of the pituitary gland: Case report and review of the literature. Neurosurgery. 1998;42(1):166-70.

19. Russell DS, Rubinstein LJ. Pathology of tumours of the nervous system. 5 th ed. Baltimore: Williams and Wilkins; 1989.

20. Smith JL, Feehery JM, O'Hara BJ, et al. Granular cell tumor of the parotid: A case report and literature review. J Ear Nose Throat. 2001;80(7):454-7.

21. Lee MW, Chang SE, Song KY, et al. S-100- negative atypical granular cell tumor: Report of a case. Int J Dermatol. 2002;41(3):168-70.

22. Lack EE, Worsham GF, Callihan MD, et al. Granular cell tumor: a clinicopathologic study of 110 patients. J Surg Oncol. 1980;13(4):30116.

23. Ordonez NG: Granular cell tumor: a review and update. Adv Anat Pathol. 1999;6(4):186-203. 